This is the author's final, peer-reviewed manuscript as accepted for publication. The publisher-formatted version may be available through the publisher's web site or your institution's library.

\title{
A wireless reflectance pulse oximeter with digital baseline control for unfiltered photoplethysmograms
}

Kejia Li, Steve Warren

\section{How to cite this manuscript}

If you make reference to this version of the manuscript, use the following information:

Li, K., \& Warren, S. (2012). A wireless reflectance pulse oximeter with digital baseline control for unfiltered photoplethysmograms. Retrieved from http://krex.ksu.edu

\section{Published Version Information}

Citation: Li, K., \& Warren, S. (2012). A wireless reflectance pulse oximeter with digital baseline control for unfiltered photoplethysmograms. IEEE Transactions on Biomedical Circuits and Systems, 6(3), 269-278.

Copyright: (C) 2011 IEEE

Digital Object Identifier (DOI): doi:10.1109/TBCAS.2011.2167717

\section{Publisher's Link:}

http://ieeexplore.ieee.org/xpl/articleDetails.jsp?tp=\&arnumber=6069825\&contentType=J ournals+\%26+Magazines\&sortType\%3Dasc_p_Sequence\%26filter\%3DAND\%28p_IS_ Number\%3A6204125\%29

This item was retrieved from the K-State Research Exchange (K-REx), the institutional repository of Kansas State University. K-REx is available at http://krex.ksu.edu 


\title{
A Wireless Reflectance Pulse Oximeter with Digital Baseline Control for Unfiltered Photoplethysmograms
}

\author{
Kejia Li, Student Member, IEEE and Steve Warren, Member, IEEE
}

\begin{abstract}
Pulse oximeters are central to the move toward wearable health monitoring devices and medical electronics either hosted by, e.g., smart phones or physically embedded in their design. This paper presents a small, low-cost pulse oximeter design appropriate for wearable and surface-based applications that also produces quality, unfiltered photo-plethysmograms (PPGs) ideal for emerging diagnostic algorithms. The design's "filter-free" embodiment, which employs only digital baseline subtraction as a signal compensation mechanism, distinguishes it from conventional pulse oximeters that incorporate filters for signal extraction and noise reduction. This results in high-fidelity PPGs with thousands of peak-to-peak digitization levels that are sampled at $240 \mathrm{~Hz}$ to avoid noise aliasing. Electronic feedback controls make these PPGs more resilient in the face of environmental changes (e.g., the device can operate in full room light), and data stream in real-time across either a ZigBee wireless link or a wired USB connection to a host. On-board flash memory is available for store-and-forward applications. This sensor has demonstrated an ability to gather high-integrity data at fingertip, wrist, earlobe, palm, and temple locations from a group of 48 subjects ( 20 to 64 years old).
\end{abstract}

Index Terms - filter-free, high-fidelity photoplethysmogram, low cost, pulse oximeter, reflectance sensor, surface biosensor, wearable, wireless.

\section{INTRODUCTION}

$\mathrm{H}$ EALTH problems such as cardiovascular disease, hypertension, diabetes, and congestive heart failure continue to plague society [1]. These conditions are primary drivers for the development of wearable and mobile health monitoring technologies that offer the potential to (a) increase the quality of life for individuals that already suffer from these health conditions and (b) prevent or mitigate the onset of disease in those that are at risk to acquire these health issues [2]. Of the array of medical devices that can be brought to bear for wearable/mobile applications that address these

Manuscript received December 3, 2010; revised April 24, 2011 and July 18, 2011; accepted August 15, 2011. This work was supported in part by the National Science Foundation under grants BES-0093916, BES-0440183, and CNS-0551626 and by the Kansas State University Targeted Excellence Program. Opinions, findings, conclusions, or recommendations expressed in this material are those of the author(s) and do not necessarily reflect the views of the NSF.

Kejia Li and Steve Warren are with the Department of Electrical \& Computer Engineering, Kansas State University, Manhattan, KS 66506, USA (kejiali@ksu.edu; swarren@ksu.edu). diseases, pulse oximeters offer significant relative promise because they provide two clinically relevant health parameters (heart rate (HR) and blood oxygen saturation $\left(\mathrm{SpO}_{2}\right)$ ), they do not require electrical contact to tissue, and they can operate at very low power [3], [4]. Additionally, the pulsatile plethysmographic data offered by this light-based sensing technique (which are usually discarded by commercial pulse oximeters after being used to calculate the parameters for the front panel display) can help to ascertain hemodynamic information that is well-suited for the assessment of the disease states listed above [5-8]. This information includes blood pressure [9], [10], arterial compliance [6], [11], [12], pulse wave velocity (PWV) [2], [13], stroke volume (and therefore cardiac output) [14], and other vascular parameters [7], [8], [15], [16]. Other relevant quantities include respiration rate [17], [18], patient motion [16], and even patient authentication [19-21].

However, low-cost pulse oximeter designs are unavailable that provide (a) quality, unfiltered PPGs ideally suitable for research and education toward the realization of new PPG diagnostics and (b) positional flexibility suitable for mobile and surface-based applications. While PPGs are often accessible from commercial desktop units via serial ports, these data have been filtered in proprietary ways to stabilize $\mathrm{HR}$ and $\mathrm{SpO}_{2}$ calculations. Further, due to their clinical prevalence, pulse oximetry and PPG analysis deserve coverage in biomedical instrumentation laboratories offered in secondary education curricula, yet low-cost pulse oximeters that provide reasonable-quality PPGs are not a staple in offthe-shelf educational kits.

Regarding ambulatory pulse oximeters, several types of wearable designs exist. Some of these use ring form factors, and others use finger clips. These designs use predominantly transmission-mode sensors. For broader use with wrist watches, head bands, socks, sensor 'Band Aids', and other wearable platforms that are unobtrusive and well suited for mobility, it makes sense to consider reflectance-mode layouts. This is especially true when one contemplates the immense potential of 'surface biosensors' (SBs): medical sensors embedded in the surface of everyday consumer electronics such as hand-held personal device assistants (PDAs), cell phones, smart phones, tablet PCs, head-mounted displays, etc. In this paradigm, physiological sensors will be accessible and signals will be easy to obtain, as human factors considerations 
for the overall product design will drive ease of use for the integrated biosensors. Additionally, each SB will utilize its host device's processor, memory, display, and wireless communication resources to provide user services typically unavailable in wearable platforms [22]. E.g., consider a reflectance pulse oximeter embedded on the back side of a cell phone alongside a built-in camera. As the user holds their finger against the reflectance sensor, the data will be processed by the microprocessor in the cell phone, and the LCD screen will display the signals and parameters.

In this domain, a reflectance sensor can employ a single small photodiode [23] as in most transmittance sensors. However, tissue is highly forward scattering, so the relative number of remitted photons detected in reflectance mode is low, yielding lower quality PPGs [5]. Improved sensor configurations are therefore often adopted to better acquire the radial reflectance profile, including a ring-shaped photodiode design [24], [25], a photodiode array around the central LEDs [26], and conversely an LED array around a central photodiode [27]. These designs generally employ cascaded high pass and low pass filters to extract the PPGs [28]. Such analog filters inevitably alter and even distort the signals of interest. These alterations are visibly obvious in some papers, and cycle-to-cycle inconsistencies can be significant. For this reason, a filter-free design is desirable. Finally, in a low-cost wearable sensor with a limited voltage range (e.g., $[0,3.3] \mathrm{V}$ if battery powered) and a low-precision analog-to-digital converter (e.g., 8 to 12 bits), maintaining a sensible vertical resolution, number of digitization levels, and sampling frequency for a PPG can be difficult without flexible baseline subtraction and PPG amplification strategies.

In summary, the desire to extract additional physiological information from PPGs acquired with reflectance-mode sensors imposes design constraints with respect to signal quality. This paper presents the design of a low-cost, wireless, reflectance-mode pulse oximeter suitable for these needs. It is initially housed on the surface of a printed circuit board but can be easily migrated to other surface-based applications. Here, a unique filter-free circuit (that digitally extracts the PPG waveform) and a two-stage, feedback-loop-driven control system enable the acquisition of unfiltered PPGs with $2^{12}$ levels of precision from varied body locations. An optimized LED/detector configuration promotes surface use, and the device signal quality and cost enhance its potential for integration into SB-based consumer devices.

\section{METHODS}

This section presents a design for a filter-free, reflectance pulse oximeter that combines many desirable features into a single platform. Implementation hardware is unspecified here; board-level components are detailed in Section III. DEVICE PROTOTYPE.

\section{A. Requirements and Device Layout}

The design requirements are outlined in Table I. Signal requirements include quality, unfiltered PPGs whose baselines are digitally removed, consistent with the discussion in
Section I. INTRODUCTION. The high sampling rate ensures that (a) primary signal and noise components are adequately sampled without aliasing and (b) secondary noise harmonics, e.g., $120 \mathrm{~Hz}$ up to several $\mathrm{kHz}$ from fluorescent lighting, are not aliased on top of the signal components of interest.

TABLE I

WiReless Reflectance PULSE OXIMETER DESIGN ReQUiREMENTS

\begin{tabular}{|c|c|}
\hline Category & Requirements \\
\hline \multicolumn{2}{|l|}{ Signal } \\
\hline Integrity & Unfiltered data with an optimal SNR \\
\hline Precision & Thousands of peak-to-peak digitization levels \\
\hline Sampling frequency & $\geq 240 \mathrm{~Hz}$ to minimize $\mathrm{PPG} /$ noise aliasing \\
\hline Baseline subtraction & Digital and filter-free \\
\hline Data availability & Full access to all pulsatile/baseline data \\
\hline \multicolumn{2}{|l|}{ Sensor } \\
\hline LED/detector geometry & $\begin{array}{l}\text { Radial arrangement, large area, and 3-5 mm } \\
\text { source/detector separation }\end{array}$ \\
\hline Ambient light operation & Adjustable gain and reference baseline \\
\hline \multicolumn{2}{|l|}{ Functionality } \\
\hline Communication & Wireless (10 $\mathrm{m}$ range) and USB \\
\hline Local storage & Onboard flash memory \\
\hline Battery & USB-rechargeable; Multi-day lifetime \\
\hline Client Software & Visualization and control panel \\
\hline \multicolumn{2}{|l|}{ Application } \\
\hline Measurement sites & $\begin{array}{l}\text { Multiple body locations; Various vascular } \\
\text { profiles and perfusion levels }\end{array}$ \\
\hline Wearability & $\begin{array}{l}\text { Low-profile reflectance layout adaptable for } \\
\text { wearable and SB applications }\end{array}$ \\
\hline Cost & Low $(\sim \$ 100)$ \\
\hline
\end{tabular}

Regarding sensor requirements, the photodetectors are ideally distributed radially around the central excitation LEDs to maximize the number of photons collected. Further, an LED/detector separation of 3 to $5 \mathrm{~mm}$ is appropriate at these wavelengths, as it maximizes the AC/DC ratio for each sensor channel, as verified experimentally [26], [29] and with Monte Carlo simulations [21]. In other words, reflectance photons that contain DC information from shallow, poorly perfused epidermal layers reflect near the central excitation LEDs and are undetected. Photons collected at greater radial distances are more likely to have traveled deeper into blood-perfused tissue and contain a greater percentage of AC data. Given the increased sensing area in a large-area detector, the control circuitry must easily compensate for baseline changes due to ambient light, tissue perfusion, respiration depth, etc.

Fig. 1 shows the block diagram for the pulse oximeter circuitry; a brief description was also included in [30]. The LED, sensor array, and operational amplifier (OPA) circuitry are coordinated by a Jennic JN5139 microcontroller. The intensity and timing of the bi-color LED are controlled by a digital-to-analog converter (DAC) and digital input/output ports (DIOs), respectively. A signal from the sensor array (four photodiodes surrounding the central bi-color LED) is first buffered and then fed to a differential OPA circuit. The buffered signal, designated here as the first-stage PPG signal (entire AC + DC contribution), is sampled by an analog-todigital converter (ADC). Another ADC collects the secondstage PPG signal (the AC portion only) from the output of the differential OPA circuit that has a positive input from another microcontroller DAC. 


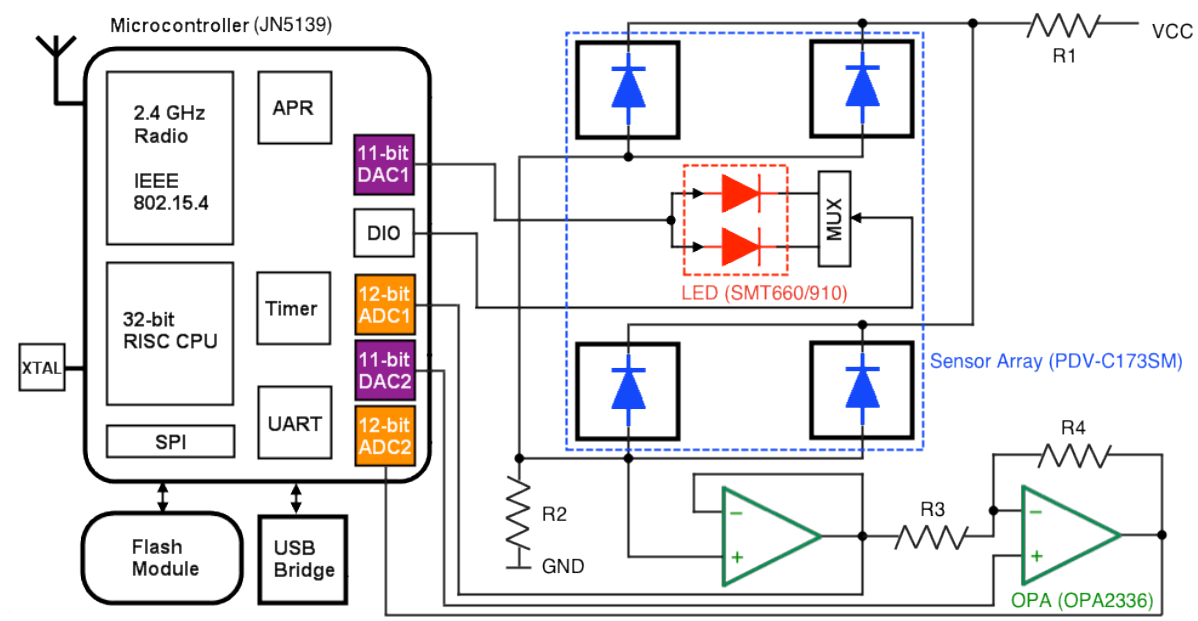

Fig. 1. Circuit-level system layout. Coordinated by the microcontroller, signal baselines are digitally extracted as an alternative to conventional filtering.

No filters are used in the signal acquisition process, whose elements will be introduced in Part C. Closed-Loop System. The battery (unstable power source) is isolated from the PPG excitation and collection circuitry, since it is powered by the microcontroller's analog peripheral regulator (APR). Normally, the pulse oximeter uses a wireless link to communicate with a receiver on a PC, and data are stored on the PC through a MATLAB graphical user interface (GUI). A mini-USB connection can provide a wired interface to the $\mathrm{PC}$ while the battery is recharged. If neither the wireless link nor the USB connection is available, sampled data will be temporarily stored on the flash memory module (e.g., for store-and-forward applications).

\section{B. AC Extraction and Drift Resistance}

The first-stage PPG is characterized by a large DC portion and a small AC portion, as in Fig. 2. The goal is to extract the second-stage AC signal by eliminating the DC component. (In many systems, a high pass filter extracts the AC signal.) If the DC portion instead remains, then obtaining hundreds to thousands of digitization levels in the AC portion over its small voltage range requires an $\mathrm{ADC}$ of very high precision (e.g., 16-bit), which is inappropriate for a low-cost, lowpower-consumption device. This extraction, or DC removal, process is executed by the OPA unit. Its role is expressed as

$$
S_{2}=G \times\left(V_{r e f}-S_{1}\right)
$$

where $S_{1}$ and $S_{2}$ are the first-stage and second-stage signals, respectively, $G$ is the gain of the OPA, and $V_{\text {ref }}$ is a userdefined reference voltage that functionally equates to the DC signal level. To show an upward-oriented PPG peak during systole as with a blood pressure curve, $V_{\text {ref }}$ is connected to the positive pin of the OPA, effectively inverting the AC signal amplitude prior to digitization.

$S_{l}$ is naturally unstable, as both its AC and DC levels are influenced by changes in intrinsic blood flow, extrinsic motion, respiration, background light, etc. These factors cause drifting in $S_{2}$. The input voltage range, or digitization range, of the 12-bit $\mathrm{ADC}$ is set to $[0,2.4] \mathrm{V}$, so one digitization level is $2.4 \mathrm{~V} / 4095$ levels $=0.586 \mathrm{mV}$. For example, given a gain $G$
$=30$ and a constant $V_{\text {ref }}$, one digitization-level increment in the DC signal results in a decrement of 30 digitization levels in $S_{2}$ according to (1). As in Fig. 2, $S_{2}$ may drift $0.3 \mathrm{~V}$ (512 digital levels) in 10 seconds, which is unacceptable because the signal will eventually clip at the lower bound of the sampling range, and clipped data mean signal corruption. To address this issue, (1) implies that one can adjust one or more elements on the right side to adjust the value of $S_{2}$ on the left. In this effort, a $V_{\text {ref }}$ adjustment is employed to resist $S_{2}$ drifting, since $V_{r e f}$ is an output of the DAC and can be easily updated. $V_{\text {ref }}$ is defined as

$$
V_{\text {ref }}=M A(t)+V_{+}
$$

where $M A(t)$, the estimator of the DC component, $V_{D C}$, is a $W$ point (e.g., $W=256$ ) moving average of the first-stage signal over the time interval that ends at $t$. $V_{+}$(the adjustable term) is added to $M A(t)$ to ensure that $V_{\text {ref }}$ makes $S_{2}$ in (1) positive.

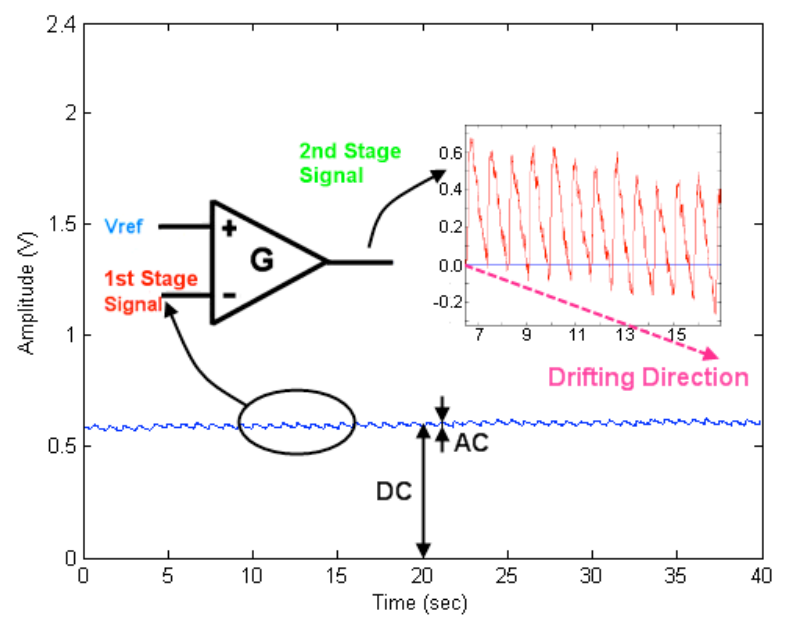

Fig. 2. A differential amplifier with gain $G$ compares the first-stage PPG $\left(S_{l}\right)$ to a DC reference voltage to obtain the second-stage PPG $\left(S_{2}\right)$.

$V_{\text {ref }}$ usually varies slowly (several seconds per digitization level change, in an environment with minimal motion and ambient noise), and the $V_{\text {ref }}$ adjustment leads to a discontinuity in $S_{2}$. Hence, the $V_{\text {ref }}$ data must also be transmitted or stored along with the digitized second-stage data in order to restore 
the original PPGs, a process called "compensation."

Fig. 3 shows a data set from the palm. Collected data are compensated to remove discontinuities caused by $V_{\text {ref }}$ jumps, or immediate value changes, in the pulsatile waveform using the following method. Inserting $V_{\text {ref }}$ from (2) into (1) and then rearranging the result isolates the first-stage signal, $S_{l}$ :

$$
S_{1}=M A(t)+V_{+}-\frac{S_{2}}{G}
$$

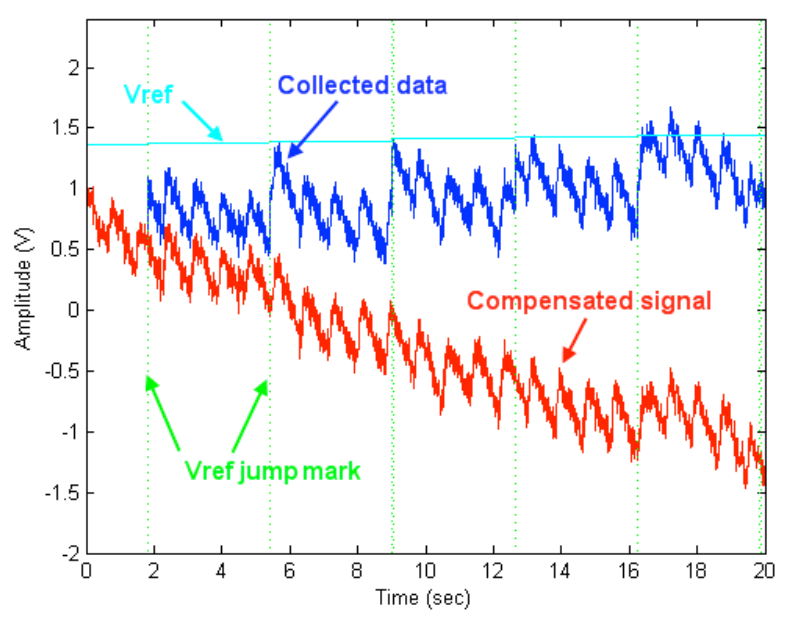

Fig. 3. Palm PPG data before (blue) and after (red) compensation.

The compensated second-stage signal, $\hat{S}_{2}$, can be represented as

$$
\hat{S}_{2}=G \times\left(V_{D C}+V_{+}-S_{1}\right)
$$

where $V_{+}$is added to $V_{D C}$ to ensure a positive $\hat{S}_{2}$. Substituting (3) into (4) yields

$$
\hat{S}_{2}=S_{2}-G \times\left(M A(t)-V_{D C}\right)
$$

Typically, $V_{D C}$ is an unknown constant, but it is sensible to initially set $V_{D C}=M A\left(t_{0}\right)$ at time $t_{0}$ and define $V_{j u m p}=M A(t)-$ $M A\left(t_{0}\right)$ at time $t\left(t>t_{0}\right)$ so that (5) becomes

$$
\hat{S}_{2}=S_{2}-G \times V_{\text {jump }}
$$

With this method, each PPG can be restored as long as the second-stage signal is unsaturated. The $V_{\text {ref }}$ adjustment effectively resists first-stage-signal drifting. For example, in Fig. 3, the compensated signal drifts below $0 \mathrm{~V}$ after 6 seconds. If no $V_{\text {ref }}$ adjustment occurs, the subsequent signal is sampled as $0 \mathrm{~V}$. To calculate blood oxygen saturation, $V_{\text {ref }}$ is usually considered equal to $V_{D C}$.

\section{Closed-Loop System}

The $V_{\text {ref }}$ adjustment mechanism not only helps to realize the AC extraction task; it also results in resilience in the PPG signal. In the control system, as illustrated in Fig. 4, two closed loops provide stability for the whole data acquisition process. The closed loop in the lower left maintains the $S_{I}$ value in a predetermined range, which is set by the Intensity Regulator that controls the led intensity via a DAC. The physical function of this control loop is to maintain the number of reflected photons at an optimal level within the active range of the photodiode, independent of a subject's vascular and perfusion profiles [30]. The closed loop in the upper right prevents $S_{2}$ from saturation, since the compensation method described in (6) requires an unsaturated second-stage signal. Upon detecting saturation onset, the Saturation Inhibitor adjusts the $V_{+}$component of $V_{\text {ref, }}$, which leads to a corresponding change in $S_{2}$ according to (1).

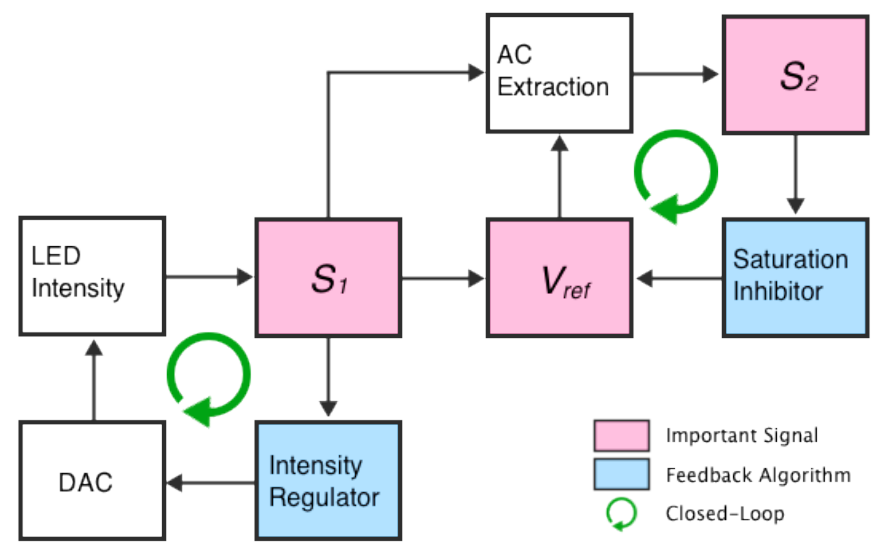

Fig. 4. Pulse oximeter control flow that illustrates how the first-stage PPG $\left(S_{l}\right)$ can be used to create the second-stage PPG $\left(S_{2}\right)$, where both signals provide feedback to stabilize the acquisition process.

To maintain signal quality, the Intensity Regulator sensitivity should be minimized. When the regulator affects changes in LED excitation level, the influence on the firststage signal converted by the photodiode sensor array will be hard to predict because the blood-perfused tissue between the LED and the sensor is an unknown system. Conversely, the sensitivity of the Saturation Inhibitor should be set high to ensure a rapid response to signal drift. Since this adjustment only influences $V_{r e}$, the native PPG is uncontaminated, and the second-stage signal can be compensated using (6).

In a controlled scenario, ambient noise variations can be ignored. If the desired signal intensity increases as the LED intensity increases, the signal-to-noise ratio (SNR) will improve. However, this implies a saturation risk due to a second-stage signal with too large of a magnitude within a fixed digitization range, in spite of the aforementioned driftresistant method. Additionally, a more intense LED consumes more power. So, an optimized intensity level should be empirically predetermined as the Intensity Regulator reference.

\section{Removable Noise}

In the U.S.A, ambient light often includes a $60 \mathrm{~Hz}$ component and the associated harmonic noise, e.g., $120 \mathrm{~Hz}$ flicker from full-wave-rectified fluorescent room lights plus higher-frequency harmonics. Most physiological information in a PPG resides in the range of $0-20 \mathrm{~Hz}$. From the NyquistShannon sampling theorem, the lowest sampling frequency, $f_{s}$, should then be $40 \mathrm{~Hz}$, but to prevent ambient noise aliasing, sampling frequencies of at least $240 \mathrm{~Hz}$ are needed.

Fig. 5 depicts the magnitude spectrum of a PPG containing ambient noise. The heart rate component is $1.329 \mathrm{~Hz}$, and its 
harmonics dominate in the frequency band below $20 \mathrm{~Hz}$. At greater frequencies, noise is apparent at $60.02 \mathrm{~Hz}, 84.43 \mathrm{~Hz}$ (unclear source), and $119.9 \mathrm{~Hz}$. Most of this noise is removable by post-processing as long as the sampling frequency is high enough that these noise components do not alias into the frequency range of the signal components of interest. Note that the raw signal exhibits a low SNR compared to PPGs from pulse oximeters that employ filters, but all signal components are intact and many can be removed to create a high SNR (see Fig. 12).

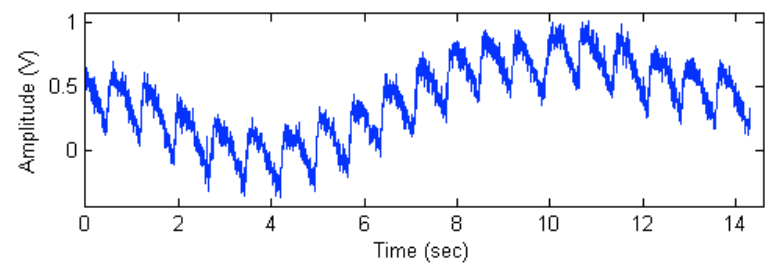

(a)

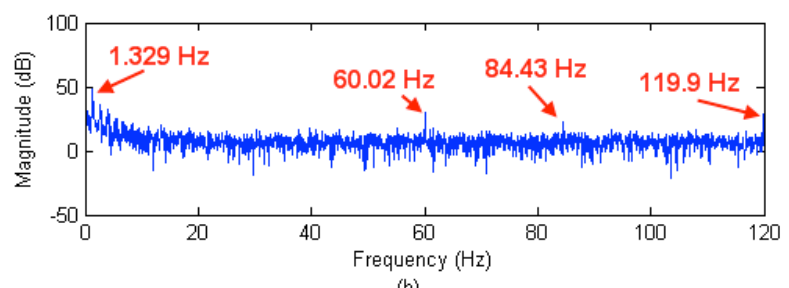

(b)

Fig. 5. An example of removable noise: (a) compensated PPG corrupted by ambient noise and (b) frequency spectrum of these data sampled at $240 \mathrm{~Hz}$.

\section{E. Motion Artifact}

Motion artifact is an issue for a pulse oximeter, especially in reflectance mode [5]. Existing literature focuses on signal processing to reduce motion artifact and restore PPGs [31]. Most methods assume that enough information exists in the corrupted signal for PPG recovery. However, if motion is severe, saturation occurs frequently and lasts for some time, leading to data loss. With this in mind, this development considered motion artifact to be a type of signal drift that can be partially addressed with a drift-resistant method ( $V_{\text {ref }}$ adjustment); the design does not address motion extraction.

Motion artifact can be classified into two categories: slight and severe. Fig. 6 demonstrates the severe condition characterized by three axes of hand motion, where the sensor is taped to the finger. Movements are within a $10 \mathrm{~cm}$ range and occur at a rate of $\sim 1 \mathrm{~Hz}$. The PPG is severely corrupted (the fundamental frequency is $1.028 \mathrm{~Hz}$ ), and it is clipped at the upper and lower bounds of the digitization range; many $\mathrm{AC}$ segments are lost and unrecoverable.

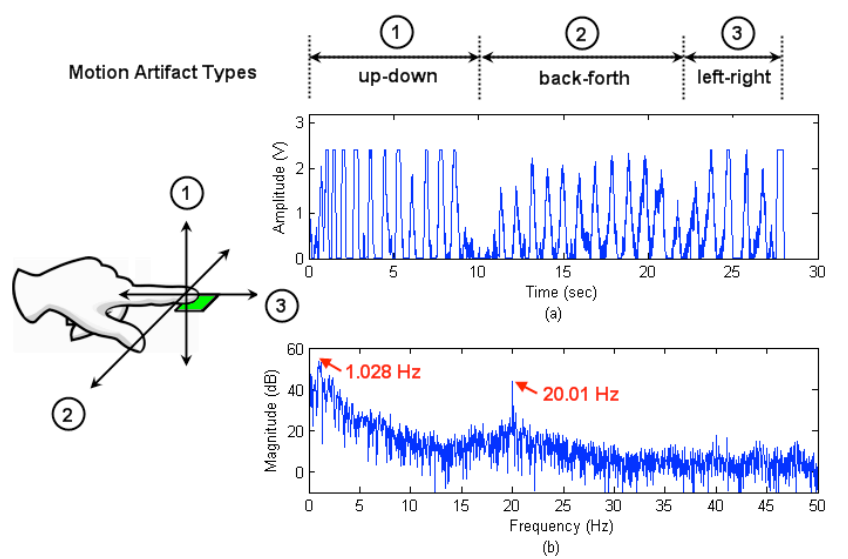

Fig. 6. (a) PPG severely corrupted by hand motion along three axes. (b) Frequency spectrum of the 28 seconds of data sampled at $100 \mathrm{~Hz}$.

The slight condition refers to, e.g., slow body movements, where a PPG retains its general shape but contains spurious components relative to a still condition. To counteract this type of artifact, the shift-resistant method is promising and relies on the setting of an optimal assignment rate and window width for $V_{\text {ref }}$ adjustment. The DAC assigns the $V_{\text {ref }}$ value to the positive amplifier pin, and that voltage remains constant until the next $V_{\text {ref }}$ assignment to the DAC. The window size of the moving average filter (the DC estimation time delay, or count) and the rate of assigning $V_{\text {ref }}$ to the DAC (not the rate of $V_{\text {ref }}$ variation) influence the second-stage signal. An extreme case occurs when the window size $W=1$ data point and the rate of assigning $V_{\text {ref }}$ to the DAC is $A=f_{s}$ : motion will never influence the signal since $M A(t) \equiv S_{1}$ and consequently $S_{2}=$ $G \times V_{+}$according to (1) and (2).

As an illustration of slight motion response, Fig. 7 shows three experimental records acquired under similar conditions (exaggerated deep respiration activity), where a different moving-average window width, $W$, and $V_{\text {ref }}$ assignment rate, $A$, is employed in each case. Only subplot (a) offers a reasonable representation of the PPG. A lower assignment rate (b) or wider window (c) causes the signal to drift severely, and some segments are nearly saturated. The empirical parameter pair $\left(W=256, A=f_{s}\right)$ was adopted for $V_{r e f}$ adjustment. 


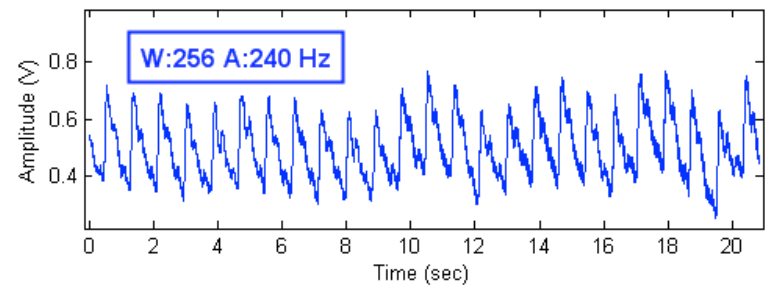

(a)

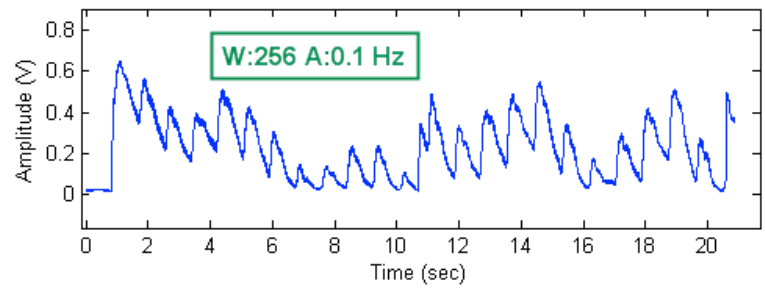

(b)

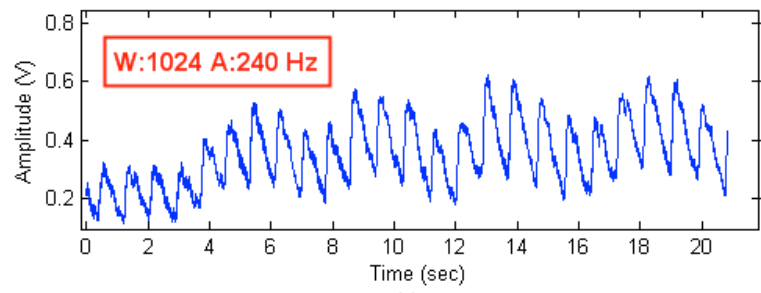

(c)

Fig. 7. Three uncompensated PPGs acquired at $f_{s}=240 \mathrm{~Hz}$ under similar slight-motion conditions but with different parameter pairs $(W, A)$.

\section{F. MATLAB Interface}

A MATLAB GUI allows a user to set/view communication parameters, visualize PPGs, process these data in real-time (e.g., digitally filter a signal with a linear-phase filter), and store raw data to files, making it a helpful development tool (see [16] for a full description). Fig. 8 illustrates an example data set obtained by this GUI, where acquisition options (e.g., Serial Port, Sampling Rate, Signal Channel, and Signal Processing Type) are specified on the left control panel. The upper axes display the raw PPG and baseline for the nearinfrared channel, whereas the lower axes show the real-time calibration coefficient, $R$, calculated from the magnitudes of the fundamental red/infrared frequency components using a Fourier transform method [21]. $R$ is updated every 0.5 seconds using the previous 4 seconds of PPG data. An overall $\mathrm{SpO}_{2}$ value is achieved by calculating the median or mean of 40 consecutive $R$ values (in a 20-second segment) and inserting the result into a pre-determined linear calibration equation.

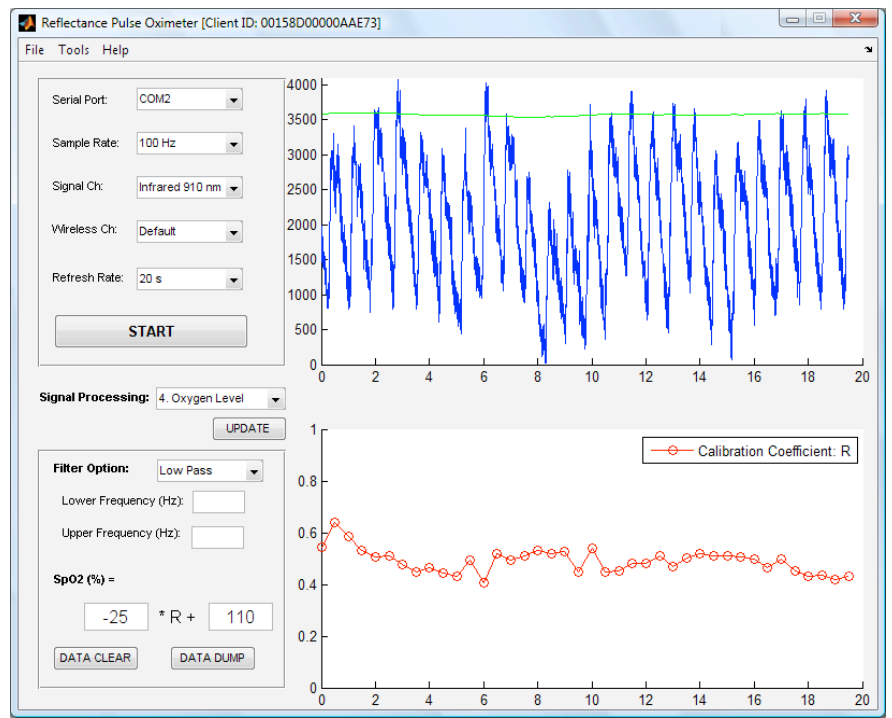

Fig. 8. Pulse oximeter MATLAB GUI. In this example, a series of calibration coefficients (lower right) is extracted from the current data (upper right).

\section{DeVice PRototype}

Figs. 9 and 10 contain top and bottom views of the pulse oximeter prototype, which consists of four main modules: microcontroller module, excitation LED module, signal sampling module, and power management module. The main printed circuit board is $41 \mathrm{~mm}$ by $36 \mathrm{~mm}$, excluding the antenna board. This hardware combines functionality from the Jennic JN5139-EK020 development kit with lessons learned from an earlier reflectance pulse oximeter design [29].

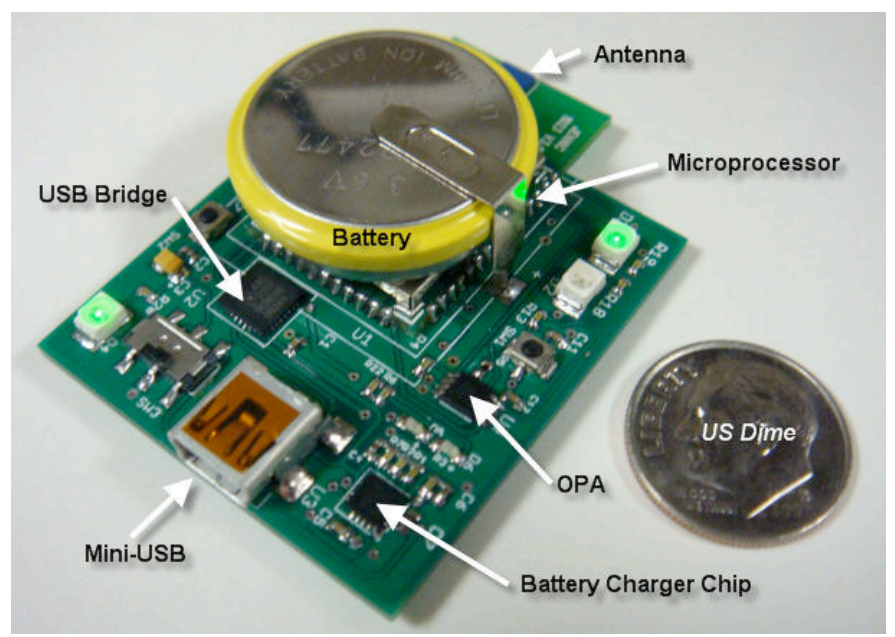

Fig. 9. Top view of the wireless reflectance pulse oximeter. 


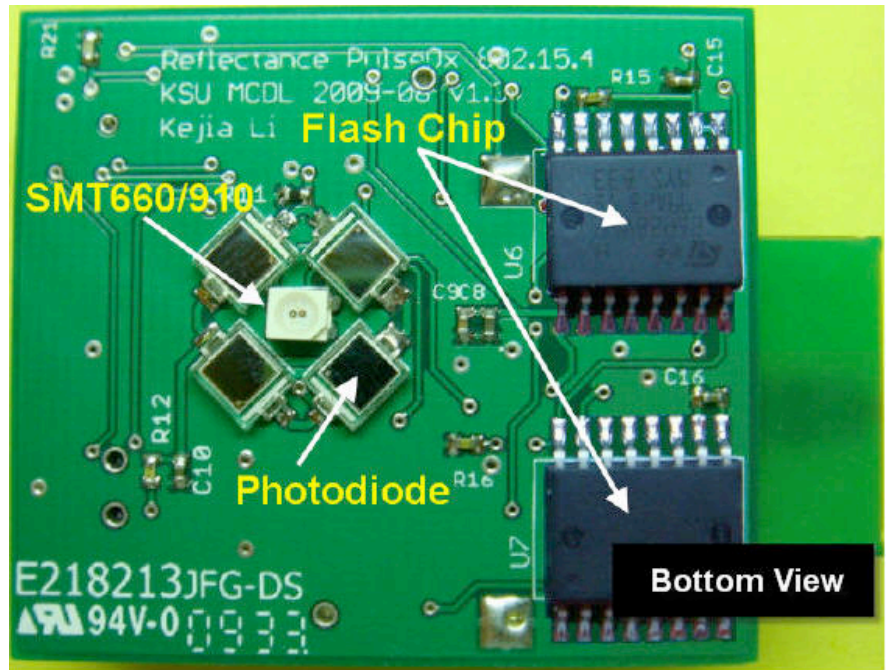

Fig. 10. Bottom view of the wireless reflectance pulse oximeter.

A microcontroller module is the prototype kernel. The JN5139 wireless module, designed for robust and secure lowpower wireless applications, integrates a 32-bit RISC processor with a 2.4 GHz IEEE 802.15.4 (ZigBee) transceiver, $192 \mathrm{kB}$ of ROM, $96 \mathrm{kB}$ of RAM, a mix of analog and digital peripherals (including four 12-bit ADCs and two 11-bit DACs), and up to 21 DIO ports. The wireless link requires the most current, with a TX (transmitter) current draw of $38 \mathrm{~mA}$ and an RX (receiver) current draw of $37 \mathrm{~mA}$. The CPU consumes $7.75 \mathrm{~mA}$ at full speed, and the current required by the peripherals (ADC, DAC, UART, Timer, etc.) is less than 1 $\mathrm{mA}$ in aggregate. The JN5139 sleep current (with an active sleep timer) is only $2.6 \mu \mathrm{A}$.

The excitation LED module uses a low-cost Marubeni SMT660/910 bi-color LED with a typical forward current of $20 \mathrm{~mA}$ and forward voltages of $1.9 \mathrm{~V}$ and $1.3 \mathrm{~V}$ for the 660 $\mathrm{nm}$ and $910 \mathrm{~nm}$ sources, respectively. The 11-bit DAC output $(0-2.4 \mathrm{~V})$ provides excitation signal modulation by managing the power supply for the excitation LED module.

The signal sampling module consists of OPA circuitry connected to the sensor array. Four API PDV-C173SM highspeed photodiodes are connected in parallel; their responsivity to wavelengths above $650 \mathrm{~nm}$ is more than $0.3 \mathrm{~A} / \mathrm{W}$. The photodiodes are arranged radially around the central LEDs and maintain a source/detector separation of 3-5 mm. The OPA chip contains two amplifier units. The sensor array signal is buffered at the first unit and amplified by the second unit.

The power management module includes two chips: (a) a Silicon Labs CP2102 USB-to-UART bridge that powers the pulse oximeter when the USB connection is detected and bridges data communication to the host and (b) an STMicroelectronics L6924D battery charger system with an integrated power switch for lithium-ion batteries which charges the battery when the USB connection is detected. An LIR2477 3.6 V lithium-ion rechargeable button cell with a capacity of 180-200 mAh serves as the power source when the USB connection is absent.

Memory chips, indicators, and buttons are also housed on the board. Two Numonyx M25PX64 64-Mbit flash memory chips with SPI bus interfaces provide storage space when the pulse oximeter works in offline mode; each consumes $20 \mathrm{~mA}$ of current while being accessed.

\section{RESUltS AND DisCUSSION}

The pulse oximeter prototypes were used to acquire hundreds of PPG records from 48 different subjects that are 20 to 64 years old. Experimental results in this section were acquired in an indoor environment utilizing the prototype pulse oximeter. The results are categorized according to conventional location (fingertip) versus other locations (wrist, earlobe, temple, etc.).

\section{A. Fingertip Data}

Fig. 11 illustrates 25 seconds of representative fingertip data from a 24-year-old subject. Both channels of PPG data, red and near-infrared, are uncompensated. The AC values of the near-infrared channel (Fig. 11 (a)) offer $1.2 \mathrm{~V}$ peak-topeak (i.e., 2048 digitization levels), and the AC values of the red channel (Fig. 11 (b)) offer fewer digitization levels: about half compared to the near-infrared channel. Even without the use of analog or digital filters, the signal demonstrates distinguishable period and amplitude information useful for $\mathrm{HR}$ and $\mathrm{SpO}_{2}$ determination. The SNRs of the raw nearinfrared and red PPGs are 8.0, and 3.0, respectively. As shown in Fig. 11 (c) and (d), up to seven harmonics reside in the spectrum of the near-infrared data (the inset shows frequency components above $5 \mathrm{~Hz}$ ), and six distinguishable harmonics reside in the spectrum of the red data. Additionally, the PPG information and noise components (e.g., $60 \mathrm{~Hz}$ and $120 \mathrm{~Hz}$ grid noise) are clearly separated in the frequency domain. To further refine the signal, a properly designed digital band pass filter can be applied. 


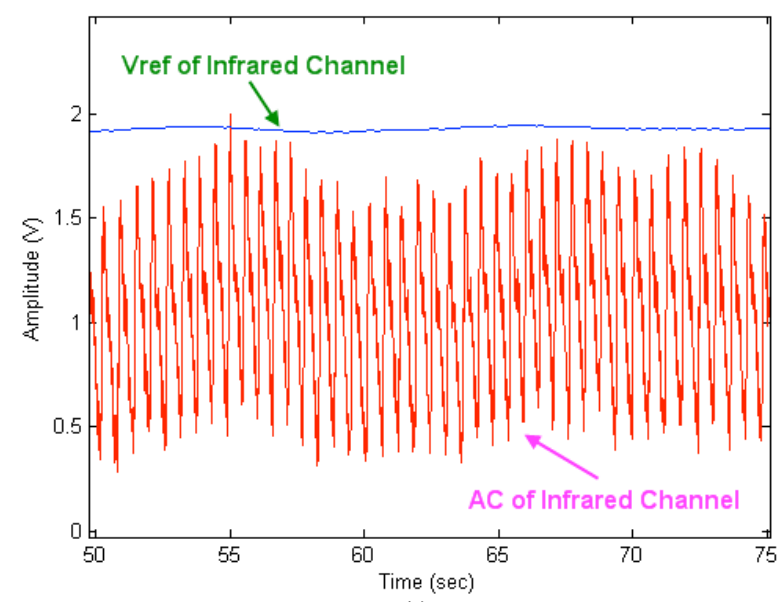

(a)

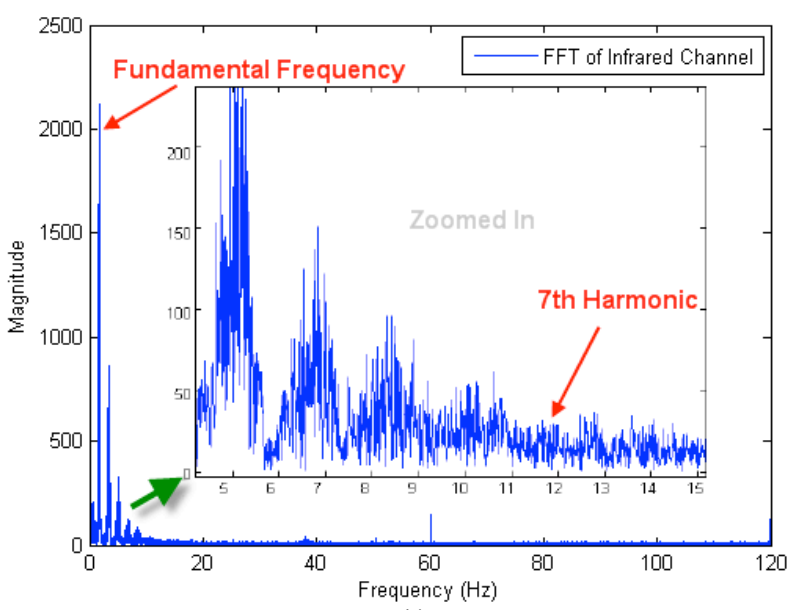

(c)

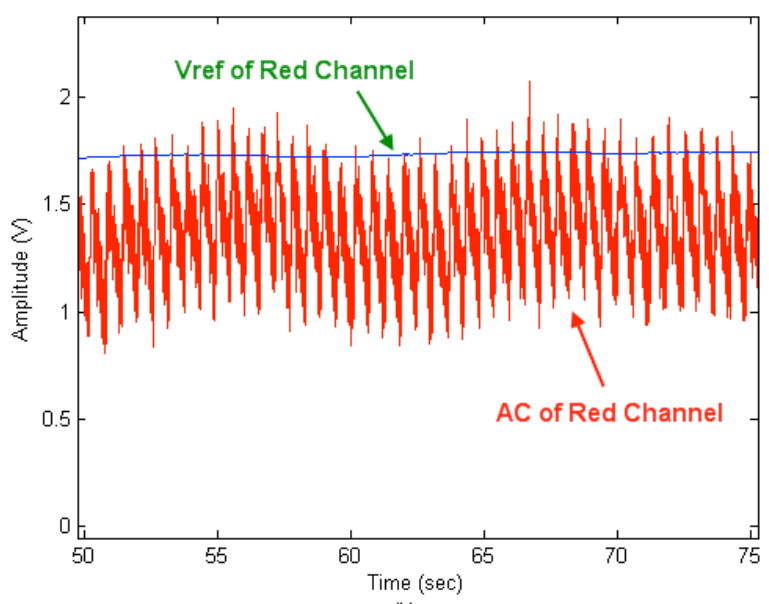

(b)

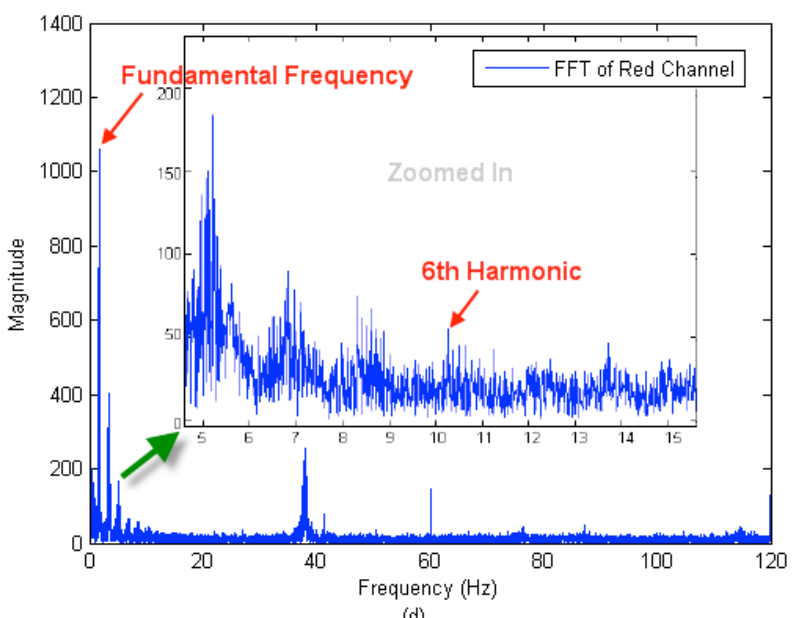

(d)

Fig. 11. Fingertip results: 25 seconds of (a) near-infrared and (b) red PPG data, accompanied by the (c) near-infrared and (d) red magnitude spectra.

Fig. 12 displays another short segment of an experimental fingertip data set, where the raw near-infrared PPG is accompanied by its real-time filtered form within a MATLAB GUI. The filter is a $200^{\text {th }}$-order low pass filter with a $10 \mathrm{~Hz}$ cut-off frequency realized by the MATLAB function firls (): a linear-phase FIR filter that uses least-squares error minimization. This high order filter causes a time delay of $t_{d}=(n-1) /\left(2 f_{s}\right)=0.414$ seconds, where $n=200$ and $f_{s}=240$ $\mathrm{Hz}$, where the time delay helps to visually separate the original and filtered waveforms. Since the peak-to-peak noise of the filtered signal is too small to be recognizable $(<1$ digitization level) the SNR is assumed to be $>2048 / 1$ if the signal amplitude is $1.2 \mathrm{~V}$. 


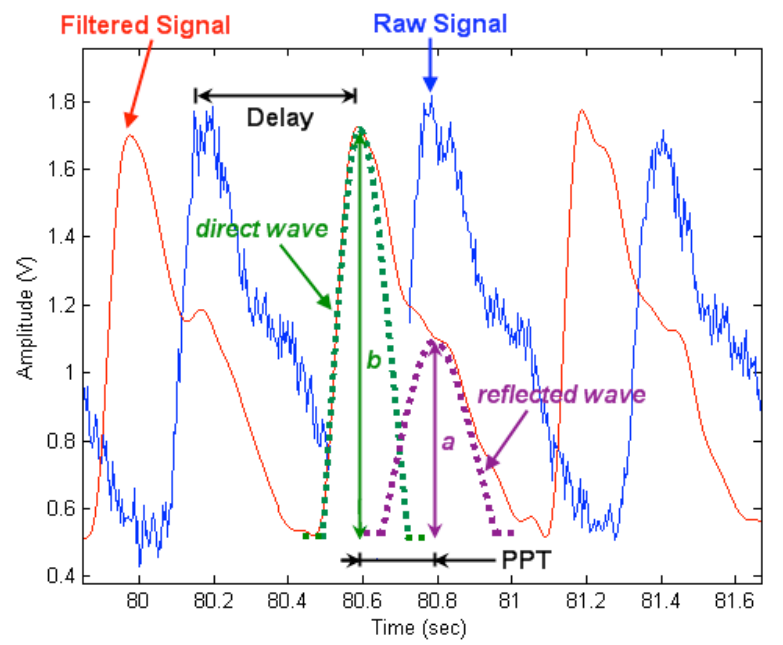

Fig. 12. Fingertip signal processing and digital volume pulse (DVP) analysis.

Digital Volume Pulse (DVP) Analysis. Cardiovascular parameters other than $\mathrm{HR}$ and $\mathrm{SpO}_{2}$ can be accurately extracted [2], [13], [15] given the quality of this DVP waveform. For example, the peak-to-peak time (PPT), as marked in Fig. 12, can be used to calculate pulse wave velocity, which correlates to arterial stiffness, and "a" and "b" are used to calculate the reflectance index, which correlates to endothelial function. Additionally, as noted in the Introduction, these unfiltered PPG waveforms could potentially lead to improved assessments of blood pressure and stroke volume via light.

\section{B. Multi-Location Data}

Fig. 13 displays experimental data from the wrist at the three placement locations depicted in Fig. 14. The signal quality in location 2 is obviously lower relative to the SNR in locations 1 and 3, but all three are suitable PPGs. At present, it is difficult to consistently obtain high quality PPG data from the wrist; that often requires the application of pressure to bring the optical sensor closer to the major arteries [21]. An operation to achieve the same effect (i.e., bending the wrist at about a $45^{\circ}$ angle), was usually employed if the PPG had a low SNR. Since subjects demonstrate a variety of different arterial locations and depths at the wrist, sensor placement flexibility is essential to acquire commendable data sets at this body location, which was also noted in [32].

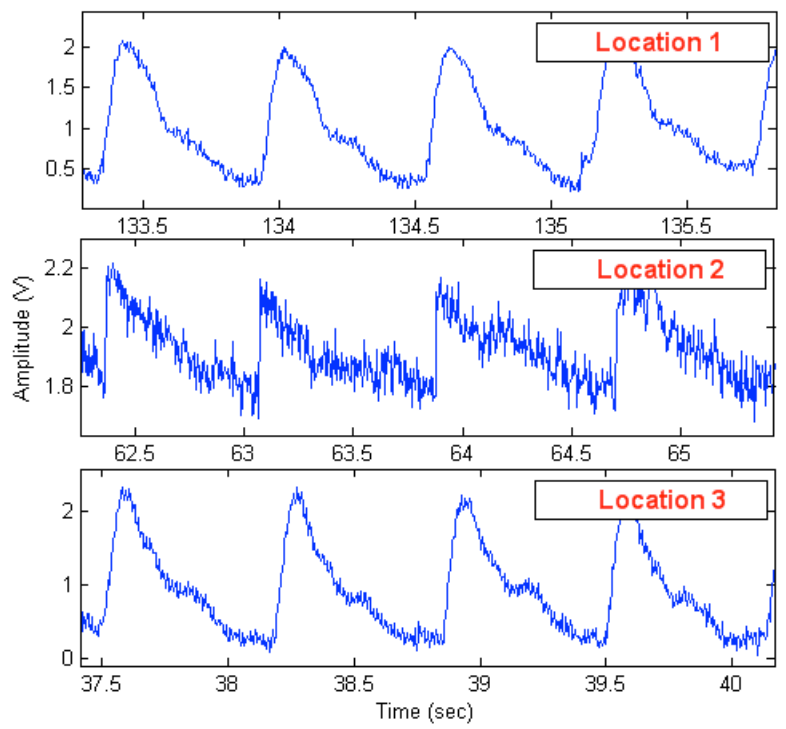

Fig. 13. Wrist PPGs corresponding to the placement locations in Fig. 14.

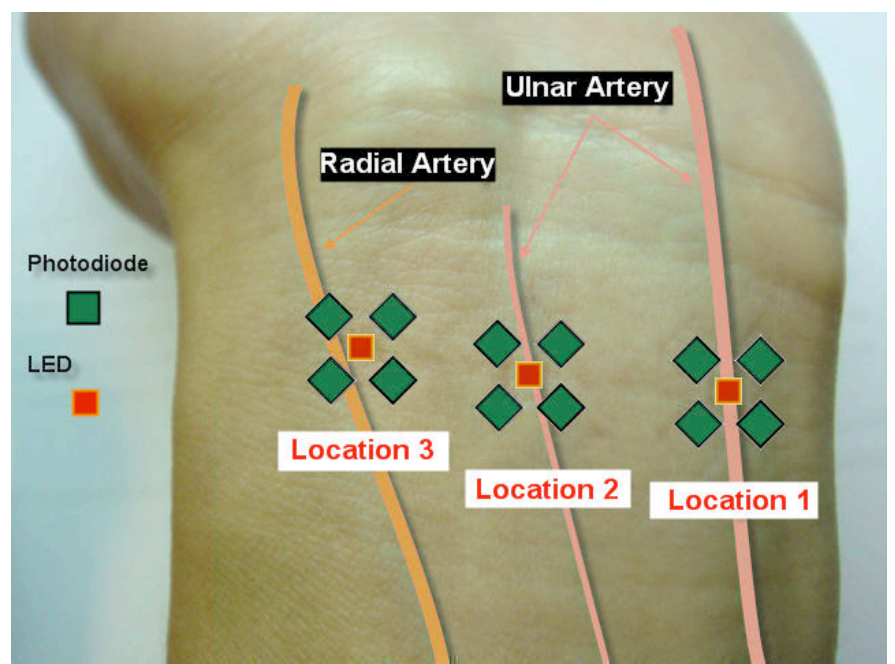

Fig. 14. Pulse oximeter measurement locations on the left wrist.

New Pulse Wave Velocity (PWV) Estimation Approach. Given the capability to acquire quality PPG data from the wrist (where the SNR dramatically improves with postfiltering), a new approach to estimate PWV has been evaluated by the authors [33]. This approach compares nearinfrared PPGs from two synchronized pulse oximeters placed at the fingertip and wrist of the same hand. PWVs can be estimated from several time differences/delays extracted from corresponding features on the two PPGs.

Fig. 15 displays two channels of data acquired from the earlobe. The near-infrared channel has an SNR of 5.7 and a peak-to-peak range of $1.0 \mathrm{~V}$ (1706 digital levels); the red channel has a much lower SNR of 1.8 and a peak-to-peak range of $0.6 \mathrm{~V}$ (1024 digital levels). 


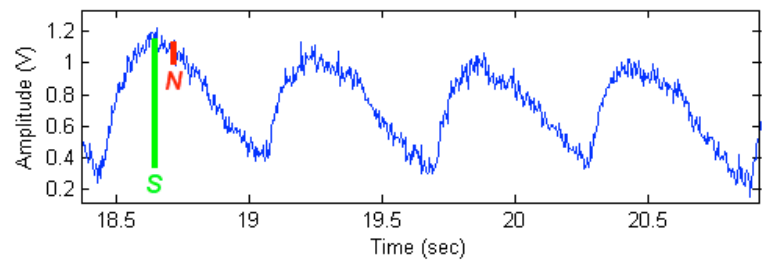

(a)

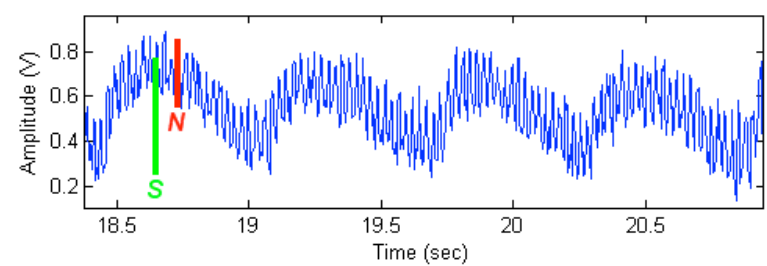

(b)

Fig. 15. Earlobe results: (a) near-infrared channel and (b) red channel.

Respiration Activity Analysis. Fig. 16 (a) displays 120 seconds of experimental data from the temple that include respiration activity and a swallowing motion. There are 33 respiration cycles present during the 120 -second recording time (i.e., the respiration rate is $0.275 \mathrm{~Hz}$ ). An FFT was applied to ascertain the visibility of these events in the magnitude spectrum, as noted in Fig. 16 (b). The peak at 1.679 $\mathrm{Hz}$ corresponds to the subject's heart rate $(100.7 \mathrm{bpm})$ and the $0.266 \mathrm{~Hz}$ frequency component is likely the respiration rate.

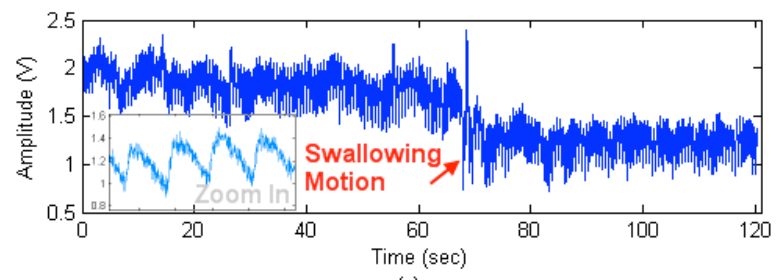

(a)

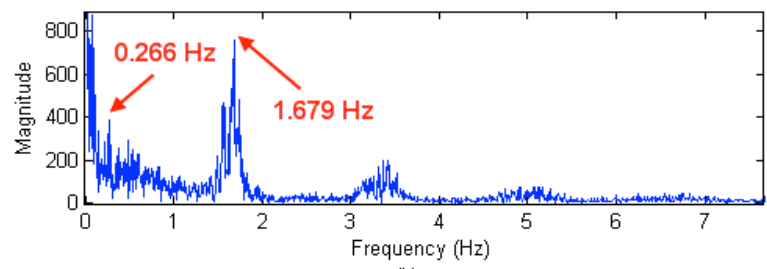

(b)

Fig. 16. Temple results: (a) time-domain PPG with respiration and swallowing motion and (b) the corresponding frequency-domain spectrum.

\section{CONCLUSION}

A high-performance wireless reflectance pulse oximeter was designed, which included functional features desired for research and education that are either unavailable or hard to find on commercial units and design optimizations based on lessons learned from previous work or published in the pulse oximetry literature. These primary features include (a) a unique filter-free circuit design, (b) full access to unfiltered PPG data, (c) many digitization levels in the pulsatile PPG signals that demonstrate a sampling frequency up to $240 \mathrm{~Hz}$, (c) a feedback mechanism to allow sensor operation in normal ambient room light, and (d) a large-area reflectance sensor that speaks to the promise of surface-infused biosensors and enables sensor placement at many body locations while optimizing the resistance of the sensor to stray photons and motion artifact. Onboard flash memory, ZigBee wireless support, and mini-USB connectivity for data transfers and battery recharging are additional highlights. The associated MATLAB GUI makes signal acquisition, visualization, restoration, and post-processing convenient.

High-integrity PPGs acquired from 48 human subjects over a wide range of ages (20 to 64 years old) indicate the device's potential as a research and teaching platform in support of the extraction of new physiological parameters from time-domain PPGs. Finally, the size, cost, layout, and design of the sensing platform speak to its suitability for wearable applications and scenarios where medical sensors are connected to or embedded in consumer electronics such as smart phones and tablet PCs.

\section{ACKNOWLEDGMENTS}

The authors thank David Huddleston, KSU Electronics Design Laboratory, for his assistance with the reflow soldering on the surface-mounted devices. Measurements from human subjects were acquired with the oversight of the Kansas State University Human Subjects Board under protocol \#5448.

\section{REFERENCES}

[1] American Heart Association, "Heart Disease and Stroke Statistics - 2009 Update At-a-Glance," 2009, http://www.americanheart.org/.

[2] S. R. Alty, N. Angarita-Jaimes, S. C. Millasseau, and P. J. Chowienczyk, "Predicting Arterial Stiffness From the Digital Volume Pulse Waveform," IEEE Transactions on Biomedical Engineering, vol. 54, pp. 2268-2275, Dec. 2007.

[3] K. N. Glaros and E. M. Drakakis, "Trade-offs for Low Power Integrated Pulse Oximeters," IEEE Biomedical Circuits and Systems Conference, Nov. 26-28, 2009, pp. 245-248.

[4] M. Tavakoli, L. Turicchia, and R. Sarpeshkar, "An Ultra-Low-Power Pulse Oximeter Implemented With an Energy-Efficient Transimpedance Amplifier," IEEE Transactions on Biomedical Circuits and Systems, vol. 4, pp. 27-38, 2010.

[5] H. Asada, P. Shaltis, A. Reisner, S. Rhee, and R. Hutchinson, "Mobile Monitoring with Wearable Photoplethysmographic Biosensors," IEEE Engineering in Medicine and Biology Magazine, vol. 22, pp. 28-40, 2003.

[6] A. Reisner, P. A. Shaltis, D. McCombie, and H. H. Asada, "Utility of the Photoplethysmogram in Circulatory Monitoring," Anesthesiology, vol. 108, pp. 950-958, May 2008.

[7] W. B. Murray and P. A. Foster, "The Peripheral Pulse Wave: Information Overlooked," Journal of Clinical Monitoring, vol. 12, pp. 365-377, Sept. 1996.

[8] K. H. Shelley, "Photoplethysmography: Beyond the Calculation of Arterial Oxygen Saturation and Heart Rate," Anesthesia \& Analgesia, vol. 105, pp. S31-S36, Dec. 2007.

[9] S. C. Millasseau, F. G. Guigui, R. P. Kelly, K. Prasad, J. R. Cockcroft, J. M. Ritter, and P. J. Chowienczyk, "Noninvasive Assessment of the Digital Volume Pulse : Comparison with the Peripheral Pressure Pulse," Hypertension, vol. 36, pp. 952-956, 2000.

[10] J. Allen and A. Murray, "Modelling the Relationship Between Peripheral Blood Pressure and Blood Volume Pulses Using Linear and Neural Network System Identification Techniques," Physiological Measurement, vol. 20, pp. 287-301, 1999.

[11] K. H. Shelley, W. B. Murray, and D. Chang, "Arterial-Pulse Oximetry Loops: A New Method of Monitoring Vascular Tone," Journal of Clinical Monitoring, vol. 13, pp. 223-228, 1997.

[12] N. Westerhof, N. Stergiopulos, and M. I. M. Noble, Snapshots of Hemodynamics, 1st ed. Boston: Springer, 2004. 
[13] S. C. Millasseau, R. P. Kelly, J. M. Ritter, and P. J. Chowienczyk, "Determination of Age-Related Increases in Large Artery Stiffness by Digital Pulse Contour Analysis," Clinical Science (London), vol. 103, pp. 371-377, Oct. 2002.

[14] J. Li, L. Yang, S. Zhang, and Y. Yang, "Computation of Cardiac Output by Pulse Wave Contour," 1st International Conference on Bioinformatics and Biomedical Engineering, Wuhan, 2007, pp. 10881090.

[15] P. J. Chowienczyk, R. P. Kelly, H. MacCallum, S. C. Millasseau, T. L. G. Andersson, R. G. Gosling, J. M. Ritter, and E. E. Änggård, "Photoplethysmographic Assessment of Pulse Wave Reflection: Blunted Response to Endothelium-Dependent Beta2-Adrenergic Vasodilation in Type II Diabetes Mellitus," Journal of the American College of Cardiology, vol. 34, pp. 2007-2014, 1999.

[16] K. Li, "Wireless Reflectance Pulse Oximeter Design and Photoplethysmographic Signal Processing," M.S. Thesis, Kansas State University, Manhattan, KS, 2010.

[17] K. Nakajima, T. Tamura, and H. Miike, "Monitoring of Heart and Respiratory Rates by Photoplethysmography Using a Digital Filtering Technique," Medical Engineering \& Physics, vol. 18, pp. 365-372, July 1996.

[18] P. A. Leonard, J. G. Douglas, N. R. Grubb, D. Clifton, P. S. Addison, and J. N. Watson, "A Fully Automated Algorithm for the Determination of Respiratory Rate from the Photoplethysmogram," Journal of Clinical Monitoring and Computing, vol. 20, pp. 33-36, 2006.

[19] L. C. Ludeman and M. I. Chacon, "Use of Blood Pulse Signature for Identity Verification," 7th International Conference on Signal Processing Applications and Technology, Boston, Massachusetts, October 7-10, 1996, pp. 1608-1612.

[20] L. C. Ludeman and M. I. Chacon, "Evaluation of Blood Pulse Signature for Potential Application in a Multisensor Biometric Identity Verification System," New Mexico State University, Final report to Sandia National Laboratories under contract AM-5506, Sept. 1995.

[21] J. T. Love, S. Warren, G. R. Laguna, and T. J. Miller, "Personal Status Monitor," Sandia National Laboratories SAND97-0418, UC-706, unlimited release, Feb. 1997.

[22] M. Locke, "Android and RTOS Together: The Dynamic Duo for Today's Medical Devices," Embedded Computing Design, pp. 36-38, April 2010.

[23] S. Rhee, B. H. Yang, and H. Asada, "Artifact-Resistant, Power-Efficient Design of Finger-Ring Plethysmographic Sensors," IEEE Transactions on Biomedical Engineering, vol. 48, pp. 795-805, July 2001.

[24] S. B. Duun, R. G. Haahr, K. Birkelund, and E. V. Thomsen, "A RingShaped Photodiode Designed for Use in a Reflectance Pulse Oximetry Sensor in Wireless Health Monitoring Applications," IEEE Sensors Journal, vol. 10, pp. 261-268, 2010.

[25] P. Branche and Y. Mendelson, "Signal Quality and Power Consumption of a New Prototype Reflectance Pulse Oximeter Sensor," 31st Annual Northeast Conference of the IEEE Bioengineering, 2-3 April 2005, 2005, pp. 42-43.

[26] Y. Mendelson and C. Pujary, "Measurement Site and Photodetector Size Considerations in Optimizing Power Consumption of a Wearable Reflectance Pulse Oximeter," 25th Annual Conference of the IEEE EMBS, 2003, pp. 3016-3019.

[27] M. Nogawa, T. Kaiwa, and S. Takatani, "A Novel Hybrid Reflectance Pulse Oximeter Sensor with Improved Linearity and General Applicability to Various Portions of the Body," 20th Annual International Conference of the IEEE EMBS, 29 Oct-1 Nov, 1998, pp. 1858-1861.

[28] D. Guowei, T. Xiaoying, and L. Weifeng, "A Reflectance Pulse Oximeter Design Using the MSP430F149," 2007 IEEE International Conference on Complex Medical Engineering, 23-27 May, 2007, pp. 1081-1084.

[29] D. Thompson and S. Warren, "A Small, High-Fidelity Reflectance Pulse Oximeter," 2007 Annual Conference and Exposition of the ASEE, Honolulu, HI, June 24-27, 2007.

[30] K. Li, S. Warren, and B. Natarajan, "Onboard Tagging for Real-Time Quality Assessment of Photoplethysmograms Acquired by a Wireless Reflectance Pulse Oximeter," IEEE Transactions on Biomedical Circuits and Systems, to be published.

[31] R. Krishnan, B. Natarajan, and S. Warren, "Two-Stage Approach for Detection and Reduction of Motion Artifacts in Photoplethysmographic Data," IEEE Transactions on Biomedical Engineering, vol. 57, pp. 1867-1876, 2010.
[32] E. Geun, H. Heo, K. C. Nam, and Y. Huh, "Measurement Site and Applied Pressure Consideration in Wrist Photoplethysmography," 23rd ITC-CSCC, 2008, pp. 1129-1132.

[33] K. Li and S. Warren, "Initial Study on Pulse Wave Velocity Acquired from One Hand Using Two Synchronized Wireless Reflectance Pulse Oximeters," 33rd Annual International Conference of the IEEE EMBS, Boston, MA, Aug. 30-Sept. 3, 2011, pp. 6907-6910.

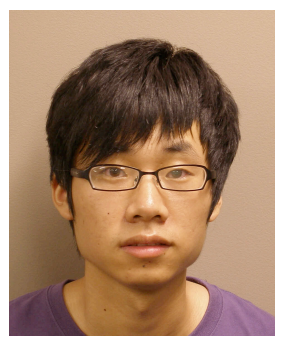

Kejia Li (S’11) received a B.S. degree in Electronic and Information Technology and Instrumentation from Zhejiang University, China, in 2008 and an M.S. degree in Electrical \& Computer Engineering from Kansas State University, USA, in 2010, where he continues to pursue a Ph.D. degree.

$\mathrm{He}$ is currently working as a Research Assistant in Medical Component Design Laboratory in the Department of Electrical and Computer Engineering at Kansas State University. His research interests involve wearable medical device design, mobile health, and statistical signal processing.

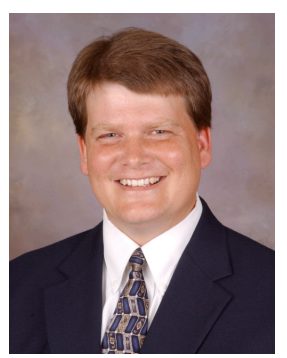

Steve Warren (M'02) received a B.S. and M.S. in Electrical Engineering from Kansas State University in 1989 and 1991, respectively, followed by a Ph.D. in Electrical Engineering from The University of Texas at Austin in 1994.

$\mathrm{He}$ is an Associate Professor in the Department of Electrical \& Computer Engineering at Kansas State University. Prior to joining KSU in 1999, Dr. Warren was a Principal Member of the Technical Staff at Sandia National Laboratories in Albuquerque, NM. He directs the KSU Medical Component Design Laboratory, a facility supported by the National Science Foundation that provides resources for the research and development of distributed medical monitoring technologies and learning tools that utilize biomedical contexts. His research focuses on (a) plug-and-play, point-of-care medical monitoring systems that utilize interoperability standards, (b) wearable sensors and signal processing techniques for the determination of human and animal physiological status, and (c) particle transport simulation applied to radiation hardness analysis of complex assemblies and the determination of photon dose distributions in tissue.

Dr. Warren is a member of the American Society for Engineering Education and the Institute of Electrical and Electronics Engineers. 